\title{
WILEY-VCH
}

DOI: 10.1002/ ((adem.201800337))

Article type: Full Paper

\section{Ultra-Low Power Corrosion Sensor Made of Iron Nanowires on Magnetic Tunnel Junctions}

Yousof Mashraei**, Selma Amara**, Zahra Albu, Jürgen Kosel*

Y. Mashraei, S. Amara, Z. Albu, J. Kosel.

Computer, Electrical and Mathematical Sciences and Engineering Division

King Abdullah University of Science and Technology (KAUST)

Thuwal 23955-6900, Saudi Arabia

*E-mail: jurgen.kosel@kaust.edu.sa@kaust.edu.sa

** The authors Y. Mashraei and S. Amara made equal contribution to the work.

Keywords: corrosion, nanowires, magnetic tunnel junction, low-power, remote sensing

Abstract

Corrosion sensors are of critical importance for monitoring the destructive potential of a corrosive environment. Due to their large surface-to-volume area, nanowires react with a remarkably high speed, rendering them attractive corrosion-sensing elements. To avoid the difficulties related to contacting nanowires, we present a magnetic approach, exploiting the fast reaction of nanowires to the environment. Iron nanowires have a high magnetization value, which decreases upon nanowire corrosion. Due to shape anisotropy, they also possess a large remnant magnetization, which is detectable by a magnetic tunnel junction sensor. Iron nanowires were fabricated by electrochemical deposition, placed on top of the magnetic tunnel junction and aligned using a magnetic field. The nanowires provide a bias field causing a change of the characteristic curve of the tunnel junction. The corrosion sensor was tested in 


\section{WILEY-VCH}

a saline solution, where the nanowires corroded, leading to a reduction of the bias field and restoration of the original characteristic. Combined, the nanowires and tunnel junction realize a highly integrated sensor concept that enables corrosion sensing with an ultra-low power consumption of less than $1 \mathrm{nW}$, a sensitivity of $0.1 \% / \mathrm{min}$, a response time of 30 minutes and a sensor area of only $128 \mu \mathrm{m}^{2}$.

\section{Introduction}

Corrosivity sensors are deployed in many industrial sectors, to prevent catastrophic failures ${ }^{[1]}$ reduce maintenance costs, ${ }^{[2]}$ or extend the life of infrastructure assets. ${ }^{[3]}$ Early detection of corrosion faults could save up to $30 \%$ of the total annual cost of corrosion; which is estimated to reach $\$ 1.372$ billion. ${ }^{[4]}$ This could be accomplished by sensors that can monitor the corrosivity of the environment to determine its impacts at an early stage. Hence, many techniques for corrosivity sensing have been developed. ${ }^{[5]}$ Especially the harsh conditions in chemical industries pose a significant threat to the facilities, causing them to corrode. ${ }^{[6-8]}$

Thin films sensors are among the preferred methods for corrosion sensing. They operate by monitoring the change in the resistance as the thin film corrodes into oxides. They are very versatile and enable a high degree of integration. More importantly, they have a fast response, due to their large surface-to-volume ratio, and they are energy efficient. ${ }^{[9-13]} \mathrm{A}$ performance leap can be expected by shrinking the sensors further, which can be implemented by employing nanowires (NWs). As a consequence of their higher surface-to-volume ratio, using NWs instead of thin films can lead to more efficient sensors. The NWs are 1D materials with very small footprints and can be cheaply mass-produced. These attributes make them great candidates for highly integrated devices with minimal power consumption and size, which are crucial aspects, for instance, in remote monitoring applications. ${ }^{[14-17]}$ However, electrical measurements on NWs are challenging, due to obvious difficulties with electrical connections. 


\section{WILEY-VCH}

Therefore, we propose a magnetic NWs corrosion sensor. Iron $(\mathrm{Fe}) \mathrm{NWs}$ in particular are very suitable for this application, since Fe has a high saturation magnetization, and in the form of NWs, the remanence magnetization retains a high value too, ${ }^{[18-22]}$ which renders them nano-sized permanent magnets. ${ }^{[23-28]}$ When exposed to a corrosive environment, they form a shell of Fe oxide around the core. The shell grows over time until the core is completely oxidized. As a consequence, the nanowires lose some of their magnetization in response to the corrosion. $^{[29,30]}$ This property of the Fe NWs is exploited for corrosion sensing and is particularly useful since most of the industrial facilities are iron-based structures. In order to detect the magnetization of the NWs, hence measure the corrosivity of the environment, the Fe NWs are integrated with a Magnetic Tunnel Junction (MTJ) sensor. The resistance of the MTJ sensor is a function of the external magnetic field, due to spin-dependent tunneling, ${ }^{[31]}$ and both high sensitivity, small size and low power consumption can be achieved. ${ }^{[32,33]}$ The NWs are placed on top of the MTJ sensor, and the corrosion of the Fe NWs is observed as a change of the MTJs resistance, when the Fe NWs start to lose their magnetization, as illustrated in Figure 1.

\section{Results and Discussion}

\subsection{Iron Nanowires}

Fe NWs are fabricated by electrochemical deposition into an alumina membrane (Figure 1S). ${ }^{[34]}$ Figure 2a shows the cross-section Scanning Electron Microscopy (SEM) image of an alumina membrane, with the electrodeposited Fe NWs inside of the pores. The NWs have an average length of $5 \mu \mathrm{m}$, and an average diameter of $45 \mathrm{~nm}$, resulting in an aspect ratio >100 (Figure 2b). This ensures a single magnetic domain in the NWs, ${ }^{[21]}$ leading to a magnetic remanence value that is about as high as the saturation value. 


\section{WILEY-VCH}

The composition of the nanowires was analyzed using Energy Dispersive X-Ray (EDX) and Raman spectroscopy. The EDX spectrum in Figure 3a shows the presence of Fe and oxygen in the NWs. The Si peak comes from the substrate (Si wafer), onto which the NWs were placed for this measurement. The presence of oxygen may indicate the presence of iron oxide, which forms spontaneously upon the release of the NWs from the membrane. ${ }^{[1]}$ Raman Spectrum analysis (Figure 3b) further elucidates the existence of the iron oxide $\left(\mathrm{Fe}_{2} \mathrm{O}_{3}\right)$ in six bands ${ }^{[35]}$. As has been shown before, ${ }^{[29]}$ in the presence of a corrosive environment, this polycrystalline structure will promote further oxidization of the NWs.

The effect of the corrosion on the magnetization of the NWs was characterized in a Superconducting Quantum Interference Device-Vibrating Sample Magnetometer (SVSM). The NWs were submerged in a saline solution of $3.5 \%$ salt concentration (typical for seawater) inside a Snap-Fit gelatin capsule. Magnetization loops of this sample were taken regularly over a period of 11 weeks. As seen in Figure 4a, the coercivity of the NWs reduced only slightly throughout the measurement period. This indicates that the switching mechanism, consisting of domain wall nucleation followed by its motion across the NW, did not change. ${ }^{[21]}$ Hence, structural integrity was not affected, and the NWs did not decompose. Another important observation from Figure 4a is the reduction of the saturation and remnant magnetizations. This is a consequence of the formation of Fe oxide, which has a lower magnetization than $\mathrm{Fe},{ }^{[29]}$ in combination with the decreasing size of the Fe core. Note, due to the experimental conditions, providing an enclosed environment, the corrosion dynamics of the encapsulated NWs is substantially slowed down. ${ }^{[36]}$ As seen in Figure $4 \mathbf{b}$, the corrosion causes a reduction in the saturation magnetization over time, which stabilizes at $45 \%$ of the original value. This indicates that the entire NWs are corroded, in agreement with earlier findings. ${ }^{[29]}$

\subsection{Magnetic Tunnel Junctions}




\section{WILEY-VCH}

The MTJ sensors of elliptical shape and $16 \times 8 \mu \mathrm{m}^{2}$ in size were fabricated using standard photolithography procedures (Figure S2). The MTJ sensors were annealed under an applied magnetic field in the direction of the long axis of the elliptic pillars; hence inducing an easy axis. Figure 5 shows the resistance measurement of an MTJ sensor at room temperature using the four-probe method. The MTJ sensor has a low resistance of $5 \Omega$ at zero field and a linear, hysteresis free dynamic range of 200e. In combination with the measurement current of $10 \mu \mathrm{A}$, the power consumption is in the order of nano Watts. The TMR ratio, which is defined as the maximum resistance change to the minimum resistance value, is $150 \%$ and the sensitivity at zero field is $3.84 \% / \mathrm{Oe}$.

\subsection{Corrosion Sensor}

The corrosion sensor consists of Fe NWs on top of an MTJ sensor. The Fe NWs were deposited and aligned on top of the MTJ sensor either along the easy axis, or perpendicular to it. The Fe NWs that are aligned along the easy axis exert an external magnetic field that is aligned or opposed the pinning field, which results in shifting the MTJ curve by 11.8 Oe. After immersing the sensor in saline solution (3.5\% salt concentration) for 2 hours, the curve shifts back by 8.1 Oe, corresponding to a loss of magnetization in the NWs, due to their corrosion (Figure 6a). On the other hand, the alignment of the Fe NWs perpendicular to the easy axis extends the dynamic range of the MTJ sensor, since their stray field applies a bias field perpendicular to the easy axis. ${ }^{[37]}$ As a consequence, the sensitivity of the MTJ sensor changes from $12.6 \% / \mathrm{Oe}$ to $0.7 \% / \mathrm{Oe}$, in exchange for a wider dynamic range that increases by 94 Oe (Figure 6b). When submersed in the saline solution for 2 hours, the corrosion of the NWs reduces their magnetization, and the sensor partially recovers its original MTJ characteristic, with a sensitivity of $2.4 \% / \mathrm{Oe}$, and a reduction of the dynamic range by $77 \mathrm{Oe}$.

An important difference between the two alignments is that in the perpendicular orientation case, a resistance change of the MTJ is apparent at zero field. From a practical 


\section{WILEY-VCH}

point of view, this is advantageous, since the corrosion measurement can be carried out without the need of applying an external magnetic field, which simplifies the device, allows for extreme miniaturization and minimizes the power consumption. Therefore, this orientation of the NWs is used for further studies of the proposed corrosion sensor.

Figure 7 shows a measurement of the corrosion sensor for 5 hours, while it was immersed in the saline solution. The corrosion sensor responded immediately at the beginning of the measurement and saturated after 2 hours, which qualitatively matches the behavior of the magnetization loss found in Figure $\mathbf{4 b}$. The values at zero field are the most important, since this is where the corrosion sensor can be operated at ultra-low power consumption. The zero-field response of the corrosion sensor is shown in (Figure 8a). We define the corrosion sensor's sensitivity $\tau$ of the environment as the slope of a linear line that connects the initial resistance value to the saturation value of the corrosion sensor,

$$
\tau=\frac{R_{\text {Sat }}-R_{0}}{t_{\text {sat }} \times R_{\text {min }}} \times 100
$$

where $\boldsymbol{R}_{\text {sat }}$ and $\boldsymbol{R}_{\boldsymbol{0}}$ are the saturation and the initial resistance values of the corrosion sensor, respectively, $\boldsymbol{t}_{\text {sat }}$ is the time it takes for the corrosion sensor to saturate, and $\mathrm{R}_{\min }$ is the minimum resistance value of the MTJ sensor.

Under the assumption that the corrosion occurs uniformly around the NWs, the rate of corrosion can also be estimated as the rate of the nanowire's oxidation across its diameter,

$$
C R=\frac{\Phi_{N W s}}{2 \times t_{s a t}}
$$

where $\mathrm{CR}$ is the corrosion rate, and $\Phi_{\mathrm{NWs}}$ is the average diameter of the NWs.

At $20{ }^{\circ} \mathrm{C}$ (Figure 8a), the sensitivity is $0.05 \% / \mathrm{min}$ and saturation is reached after 2 hours; thus, the corrosion rate is $0.1 \mathrm{~mm} /$ year. In order to study the effect of different environmental corrosivities, the temperature of the saline solution was varied and the rate of 


\section{WILEY-VCH}

change of the resistance was determined. Figure $\mathbf{8 b}$ shows the effect of the temperature of the saline solution on three different corrosion sensors. At a temperature of $40{ }^{\circ} \mathrm{C}$, the corrosion is accelerated with $0.07 \% / \mathrm{min}$ and the full corrosion is achieved after 1 hour, implying a corrosion rate of $0.2 \mathrm{~mm} / \mathrm{year}$. Similarly, at $60{ }^{\circ} \mathrm{C}$; the corrosion increased to $0.1 \% / \mathrm{min}$ and saturated after 30 minutes, with a corrosion rate of $0.4 \mathrm{~mm} / \mathrm{year}$. Hence, the corrosion rates increase with temperature, with a factor of 1.4 every $20{ }^{\circ} \mathrm{C}$ increment in temperature. This nonlinear increase is due to the oxide formation, and the oxygen solubility dependence on temperature. ${ }^{[38,39]}$

The responsive behavior of the corrosion sensor is very similar to the Fe NWs corrosion characteristic found with the SVSM (Figure 4). Their change in remanence magnetization is inherently transferred into the corrosion sensor performance. Since the NWs can fully be corroded within 30 minutes, they provide a quick measure of the environment's corrosivity. The high sensitivity of the corrosion sensor is facilitated by the sensitive MTJ sensor, which is capable of detecting the minute changes in the NWs magnetic field.

\section{Conclusion}

In this work, a magnetic NW corrosion sensor is presented that exploits the high magnetic remanence and the large surface-to-volume ratio of the Fe NWs together with the sensitivity and power efficiency of MTJs. Upon the Fe NWs corrosion, their stray magnetic field is weakened, which is detected using a simple resistance measurement of an MTJ sensor. Two different NW alignments were studied, i.e., parallel to the easy and hard axes of the MTJ sensor. While both configurations reflect the corrosion of the NWs in the characteristic curves of the sensors, the alignment perpendicular to the easy axis is preferable, since it shows a dependence of the MTJ's resistance to the corrosion at zero magnetic field. The corrosivity of the environment was estimated by evaluating the corrosion rate of the Fe NWs. The integration of the MTJ and NWs, and the operation at zero magnetic field, resulted in a sensor 


\section{WILEY-VCH}

with ultralow power consumption of less than $1 \mathrm{nW}$, a fast response of 30 minutes in saline solution and a small size of only $128 \mu \mathrm{m}^{2}$. The sensitivity of the corrosion sensor reacted to temperature changes with a sensitivity of $0.07 \% / \mathrm{min}$ and a corrosion rate of $0.4 \mathrm{~mm} / \mathrm{year}$.

\section{Experimental Section}

The Fe NWs were fabricated using the well-known electrodeposition into porous anodic alumina disk technique (see the supporting information section). A sample of about $100 \times 10^{6} \mathrm{Fe}$ NWs was added to a Snap-Fit gelatin capsule and characterized in the SVSM (Quantum Design, MPMS®3). The magnetic field was swept between $\pm 1 \mathrm{~T}$, and the temperature was maintained at $20{ }^{\circ} \mathrm{C}$. Then a $50 \mu \mathrm{L}$ drop of a saline solution of $3.5 \%$ salt concentration, was added to the capsule with the Fe NW sample and enclosed, trapping an air bubble of $150 \mu \mathrm{L}$ inside the capsule. The sample was then characterized under the same conditions for 11 weeks.

The MTJ sensors were fabricated in-house using standard photolithography (Figure S2). The sensors were then annealed under vacuum in an applied magnetic field of $0.8 \mathrm{~T}$ along the long axis of the MTJ at $360{ }^{\circ} \mathrm{C}$ for 2 hours, and then cooled down for 4 hours. This induces an easy axis along the long axis of the MTJ sensor.

The Fe NW alignment on top of the MTJ sensor was carried out by applying an external magnetic field of 100 Oe in the desired direction and adding a $1 \mu \mathrm{L}$ drop of ethanol containing well-dispersed Fe NWs of $6.6 \mu \mathrm{g} / \mathrm{mL}$ (Figure S3). Once the ethanol drop was normally dried, the sensor was imaged in the SEM to check the presence of the Fe NWs on top of the MTJ sensor.

The measurement setup consists of Helmholtz electromagnetic coils and a probe station. The coils were driven by a Keithley $2400-\mathrm{C}$ in current source mode, and the current was amplified through a Kepco Bop72-6m bipolar amplifier. The sensors were probed and characterized using a Keithley $2400-\mathrm{C}$ in resistance measurement mode. The magnetic field 


\section{WILEY-VCH}

was monitored by using a F.W. Bell Gaussmeter model 6010, and regulated via a LabView program. All resistance measurements by the Keithley $2400-\mathrm{C}$ were done under the conditions of limiting the supplied current to $10 \mu \mathrm{A}$, and the maximum compliance voltage of $0.1 \mathrm{~V}$. The magnetic field is applied in the direction of the easy-axis of the MTJ, Figure S4.

\section{Supporting Information}

Supporting Information is available from the Wiley Online Library or from the author.

\section{Acknowledgements}

Research reported in this publication was supported by the King Abdullah University of Science and Technology (KAUST). The authors also thank the KAUST Nanofabrication, imaging and characterization core laboratory staff at KAUST.

Received: ((will be filled in by the editorial staff))

Revised: ((will be filled in by the editorial staff))

Published online: ((will be filled in by the editorial staff)) 


\section{WILEY-VCH}

\section{References}

[1] Z. C. Petrović, Military Technical Courier/Vojnotehnicki Glasnik 2016, 64.

[2] T. Hoar, "Review Lecture-Corrosion of metals: its cost and control", presented at Proc. R. Soc. Lond. A, 1976.

[3] J. Smith, Y. P. Virmani, Turner-Fairbank Highway Research Center, 2000.

[4] G. Jacobson, G. Koch, O. Moghissi, D. Kroon, NATL ASSOC CORROSION ENG 1440 SOUTH CREEK DRIVE, HOUSTON, TX 77084-4906 USA, 2016.

[5] V. S. Agarwala, P. L. Reed, S. Ahmad, "Corrosion detection and monitoring-A review", presented at CORROSION, $\mathbf{2 0 0 0 .}$

[6] M. Finšgar, J. Jackson, Corrosion Science 2014, 86, 17.

[7] B. Sherar, I. Power, P. Keech, S. Mitlin, G. Southam, D. Shoesmith, Corrosion Science 2011, 53, 955.

[8] M. H. Dorsey, B. J. Saldanha, N. H. Sridhar, L. H. Yang, D. R. Demarco, G. A. Fisher, CORROSION, 2005.

[9] M. H. Dorsey, L. Yang, N. Sridhar, CORROSION, 2004.

[10] R. Feng, M. Ziomek-Moroz, P. R. Ohodnicki, "Fe Thin Film Coated Optics for Corrosion Monitoring", presented at Applied Industrial Optics: Spectroscopy, Imaging and Metrology, 2017.

[11] L. Yang, N. Sridhar, CORROSION/2006, paper 2006.

[12] D. Chen, M. Yen, P. Lin, S. Groff, R. Lampo, M. McInerney, J. Ryan, Materials 2014, $7,5746$.

[13] D. Mizuno, S. Suzuki, S. Fujita, N. Hara, Corrosion Science 2014, 83, 217.

[14] L. Guo, N. Xie, C. Wang, X. Kou, M. Ding, H. Zhang, Y. Sun, H. Song, Y. Wang, G. Lu, Sensors and Actuators B: Chemical 2018, 255, 1015.

[15] K. G. Nikolaev, V. Maybeck, E. Neumann, S. S. Ermakov, Y. E. Ermolenko, A. Offenhäusser, Y. G. Mourzina, Journal of Solid State Electrochemistry 2018, 22, 1023. 


\section{WILEY-VCH}

[16] D. Zhou, X. Cao, Z. Wang, S. Hao, X. Hou, F. Qu, G. Du, A. M. Asiri, C. Zheng, X. Sun, Chemistry-A European Journal 2017, 23, 5214.

[17] F. Patolsky, C. M. Lieber, Materials today 2005, 8, 20.

[18] Z. Ai, Z. Gao, L. Zhang, W. He, J. J. Yin, Environmental science \& technology 2013, $47,5344$.

[19] A. Jagminas, K. Mažeika, J. Reklaitis, M. Kurtinaitienė, D. Baltrūnas, Materials Chemistry and Physics 2008, 109, 82.

[20] B. Kalska-Szostko, U. Wykowska, K. Piekut, E. Zambrzycka, Colloids and Surfaces A: Physicochemical and Engineering Aspects 2013, 416, 66.

[21] Y. P. Ivanov, M. Vázquez, O. Chubykalo-Fesenko, Journal of Physics D: Applied Physics 2013, 46, 485001.

[22] H. Cao, Z. Xu, D. Sheng, J. Hong, H. Sang, Y. Du, Journal of Materials Chemistry 2001, 11, 958 .

[23] M. A. Khan, A. Alfadhel, J. Kosel, IEEE Transactions on Magnetics 2016, 52, 1.

[24] A. I. Martínez-Banderas, A. Aires, F. J. Teran, J. E. Perez, J. F. Cadenas, N. Alsharif, T. Ravasi, A. L. Cortajarena, J. Kosel, Scientific reports 2016, 6, 35786.

[25] A. Alfadhel, M. A. Khan, S. C. de Freitas, J. Kosel, IEEE Sensors Journal 2016, 16, 8700.

[26] O. Yassine, A. Zaher, E. Q. Li, A. Alfadhel, J. E. Perez, M. Kavaldzhiev, M. F. Contreras, S. T. Thoroddsen, N. M. Khashab, J. Kosel, Scientific reports 2016, 6, 28539.

[27] A. Alfadhel, J. Kosel, Advanced Materials 2015, 27, 7888.

[28] M. F. Contreras, R. Sougrat, A. Zaher, T. Ravasi, J. Kosel, International journal of nanomedicine 2015, 10, 2141.

[29] Y. P. Ivanov, A. Alfadhel, M. Alnassar, J. E. Perez, M. Vazquez, A. Chuvilin, J. Kosel, Scientific reports 2016, 6, 24189. 


\section{WILEY-VCH}

[30] S. M. Sakhamuri, S. P. K. Gummadi, R. C. Toonen, O. R. Camacho, "Corrosivity sensor based on metallic nanowires", presented at SENSORS, 2016 IEEE, 2016.

[31] S. S. Parkin, C. Kaiser, A. Panchula, P. M. Rice, B. Hughes, M. Samant, S.-H. Yang, Nature materials 2004, 3, 862.

[32] A. Jander, C. Smith, R. Schneider, "Magnetoresistive sensors for nondestructive evaluation", presented at Proc. SPIE, 2005.

[33] M. Pannetier, C. Fermon, G. Le Goff, J. Simola, E. Kerr, Science 2004, 304, 1648.

[34] J. W. Diggle, T. C. Downie, C. Goulding, Chemical Reviews 1969, 69, 365.

[35] M. Krajewski, K. Brzozka, W. Lin, H. Lin, M. Tokarczyk, J. Borysiuk, G. Kowalski, D. Wasik, Physical Chemistry Chemical Physics 2016, 18, 3900.

[36] H. Uhlig, D. Triadis, M. Stern, Journal of the Electrochemical Society 1955, 102, 59.

[37] X. Liu, C. Ren, G. Xiao, Journal of Applied Physics 2002, 92, 4722.

[38] M. Bert, Materials Technology Institute, St. Louis 2008, 2015.

[39] W. Vernon, Transactions of the Faraday Society 1935, 31, 1668. 


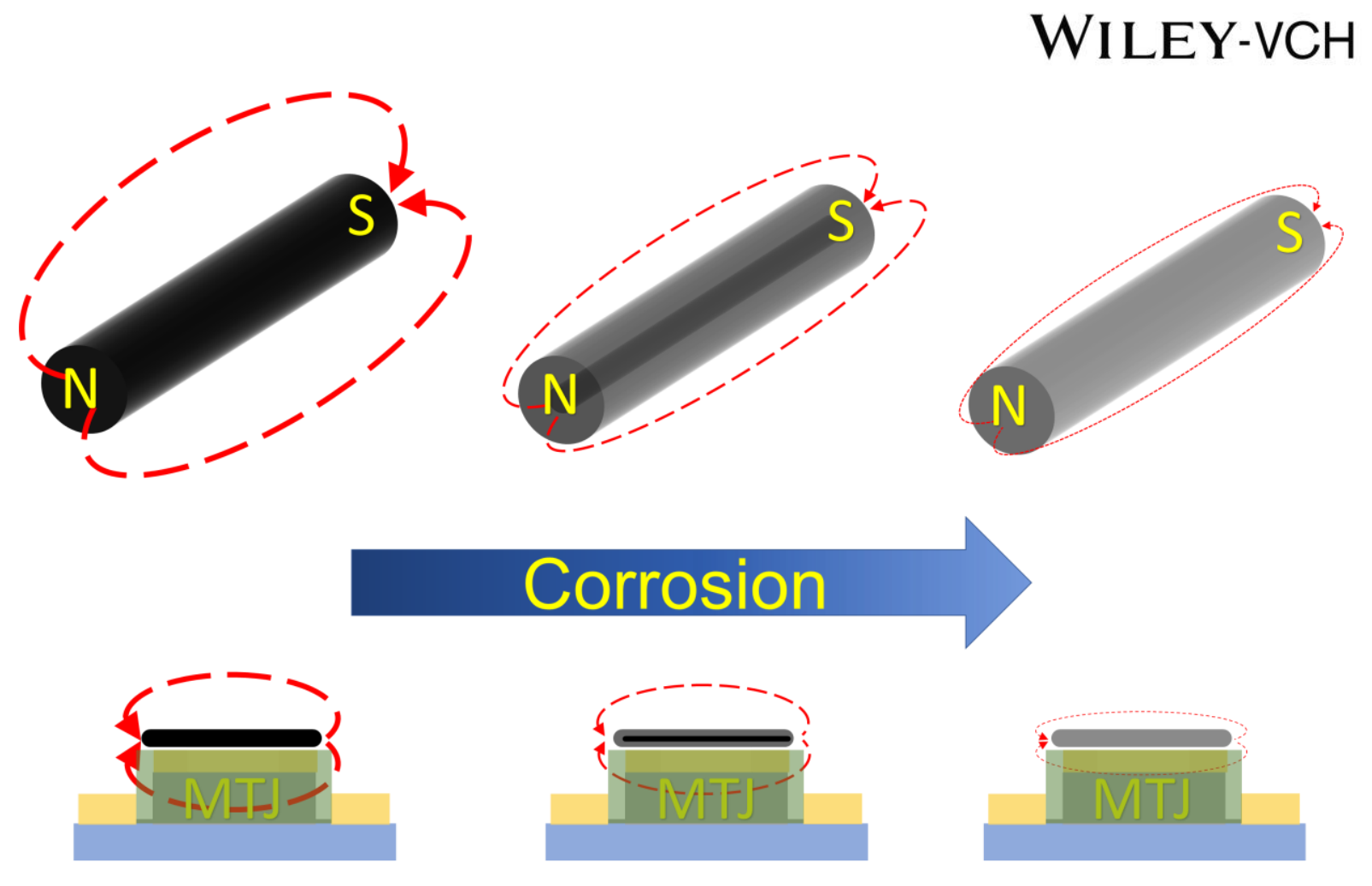

Figure 1. Illustration of the magnetic NW corrosion sensor concept. When the Fe NWs start to corrode, their magnetization decreases, which is detected by the MTJ sensor. 


\section{WILEY-VCH}
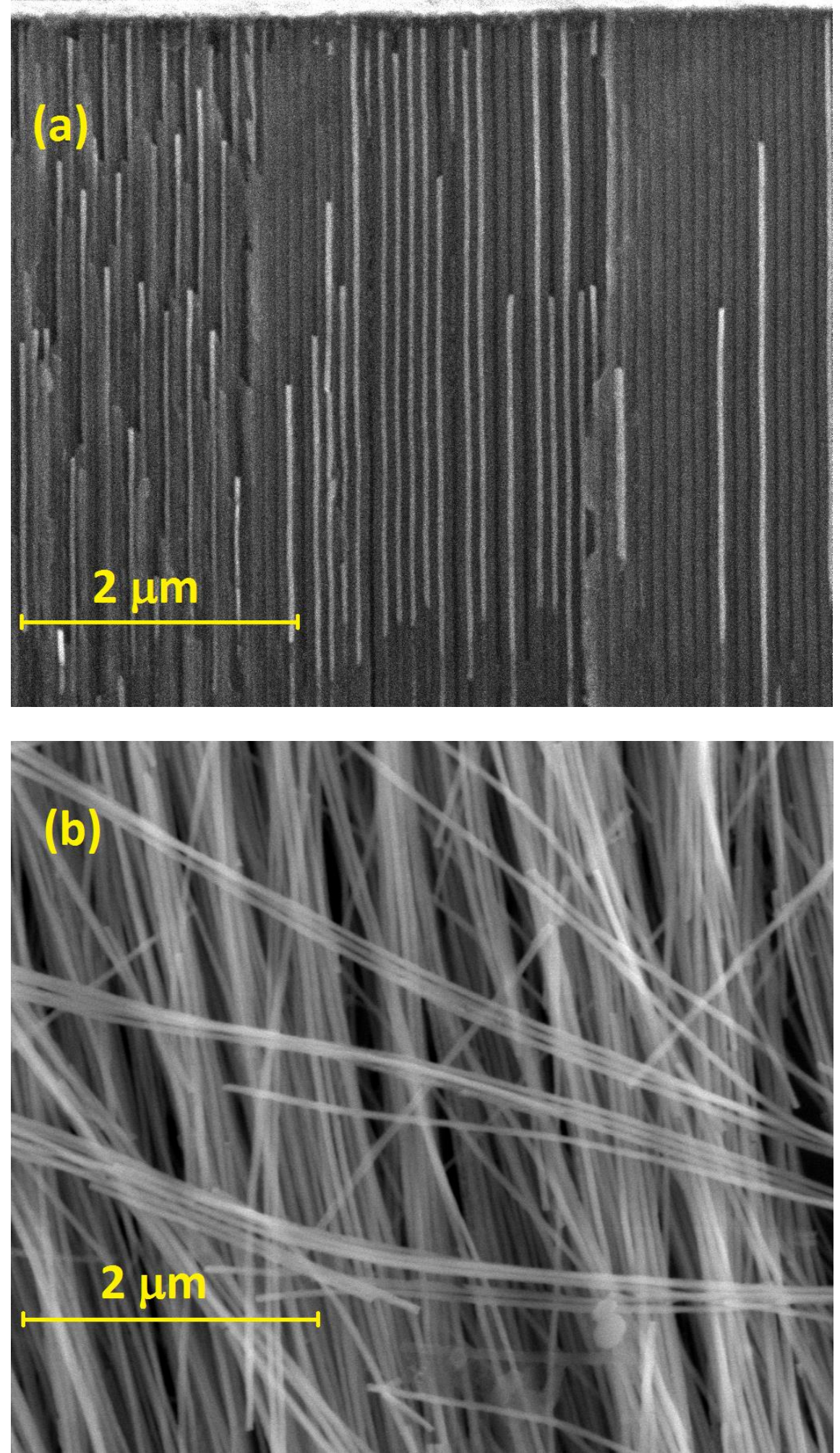

Figure 2. SEM images of the electrodeposited Fe NWs in the alumina membrane (a) and after release (b). 
(a)

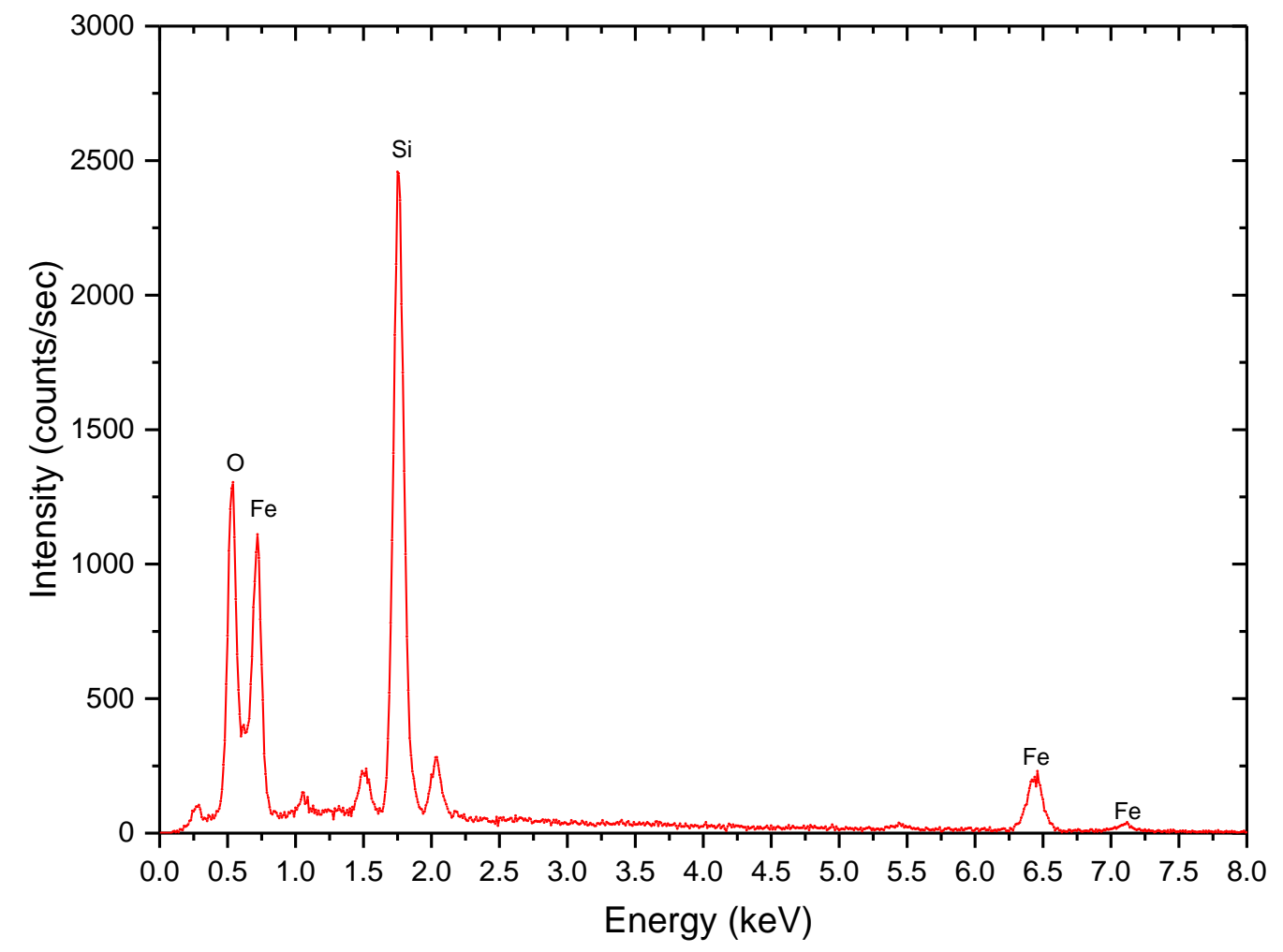

(b)

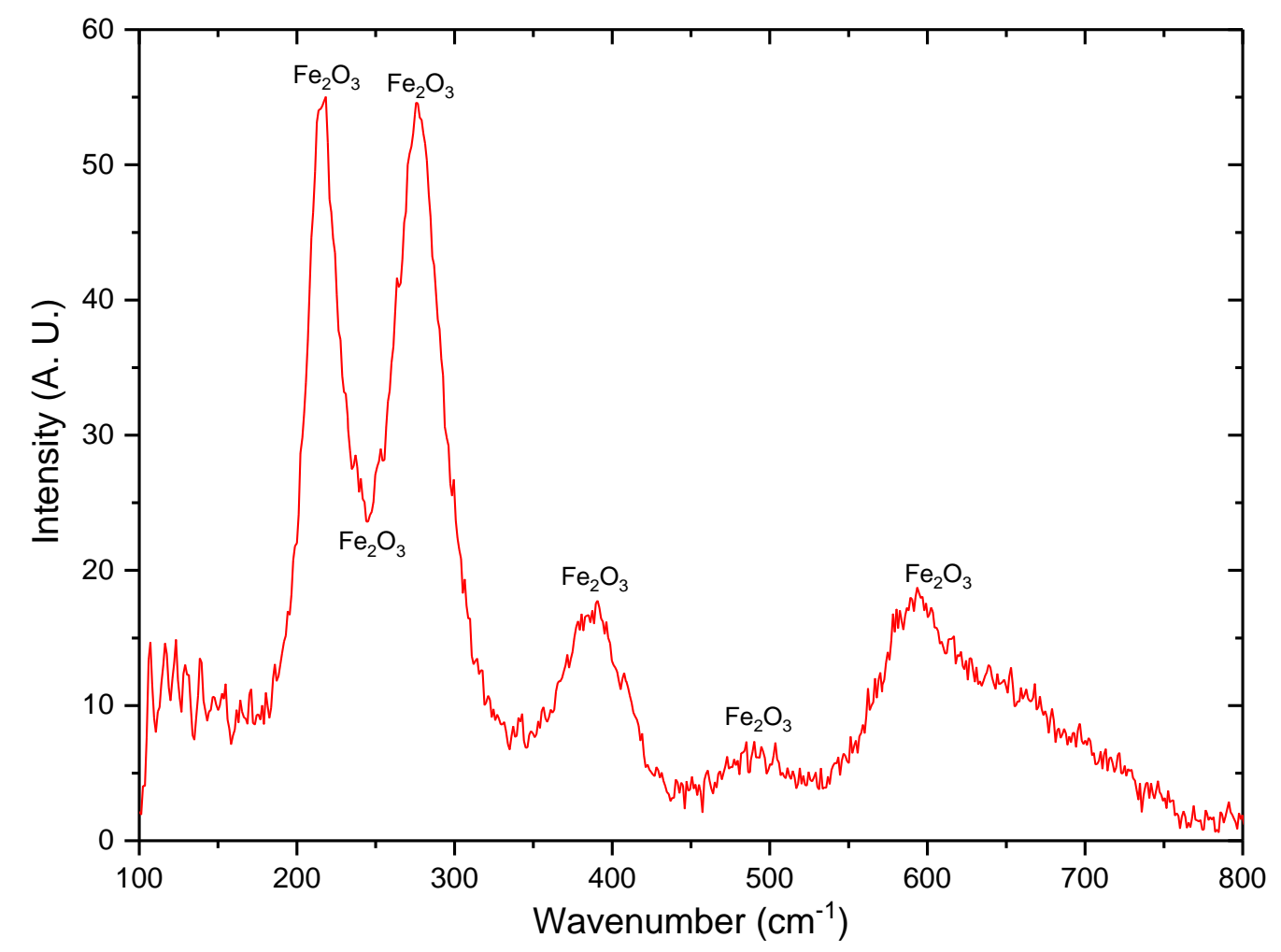

Figure 3. (a) EDX spectrum and (b) Raman spectrum of the Fe NWs. 
(a)

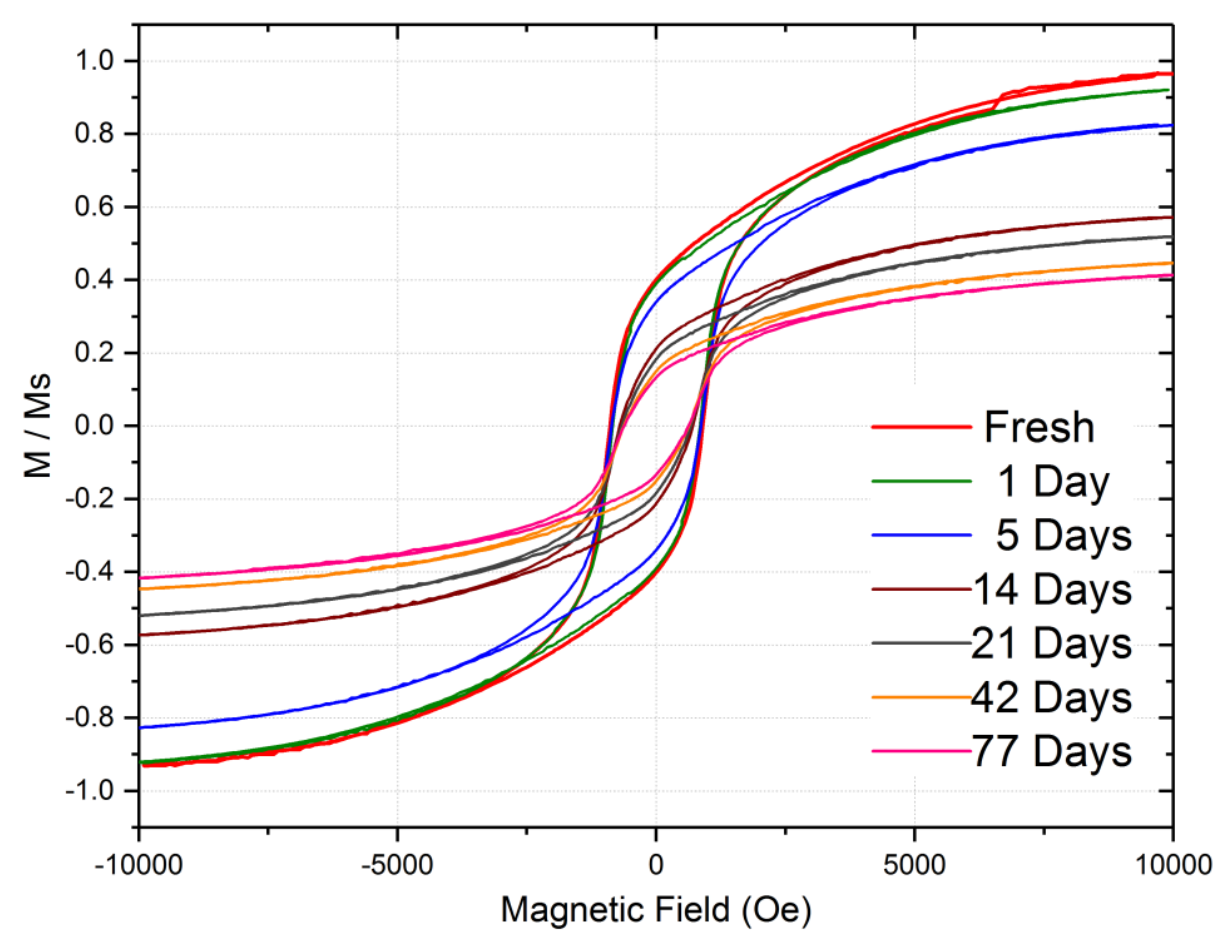

(b)

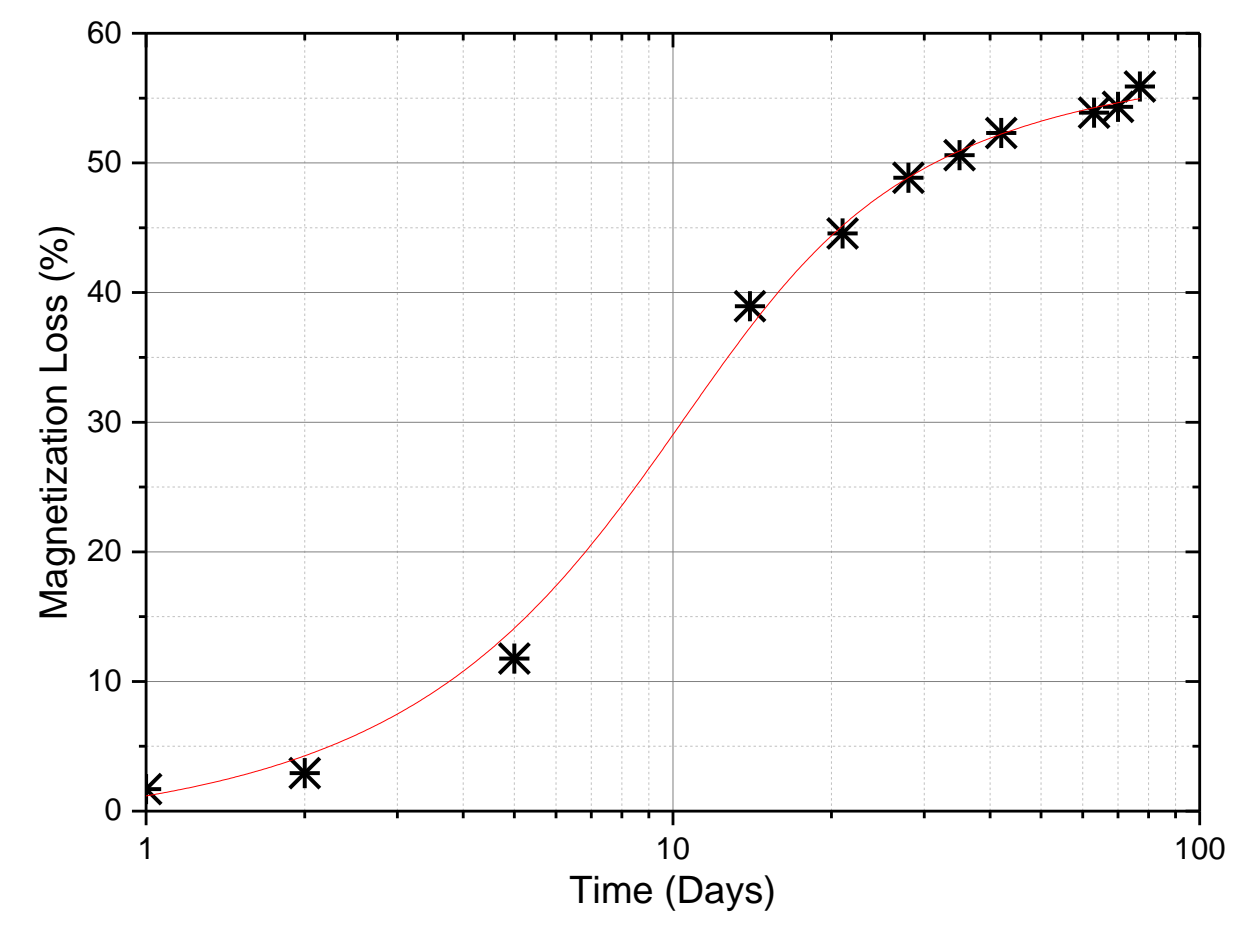

Figure 4. (a) Magnetization loops obtained by SVSM and (b) saturation magnetization of Fe NWs in saline solution over a period of 11 weeks. 
WILEY-VCH

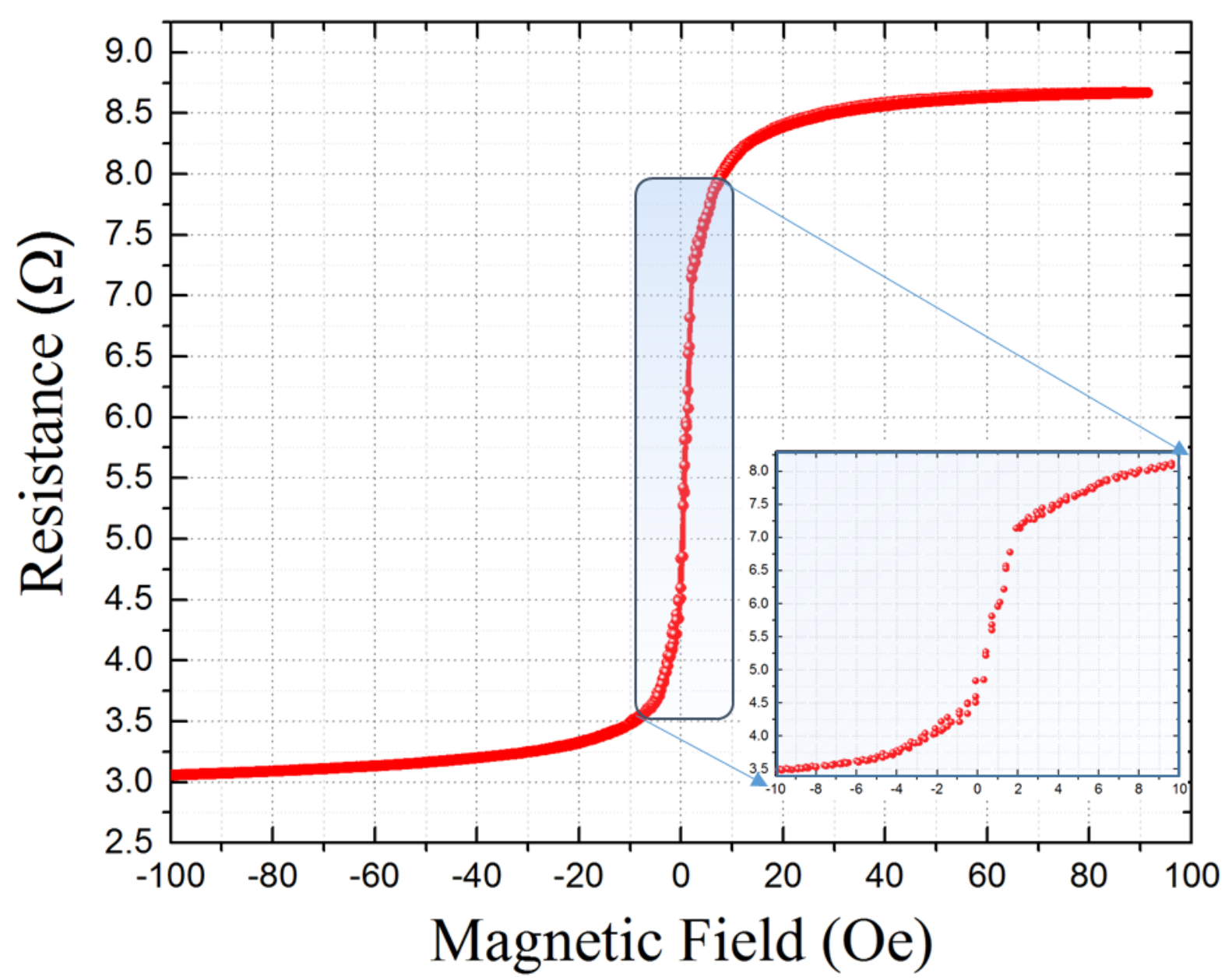

Figure 5. Characteristic curve of an MTJ sensor of elliptical shape $\left(16 \times 8 \mu \mathrm{m}^{2}\right)$ and zoom-in to the zero-field range. 


\section{WILEY-VCH}

(a)

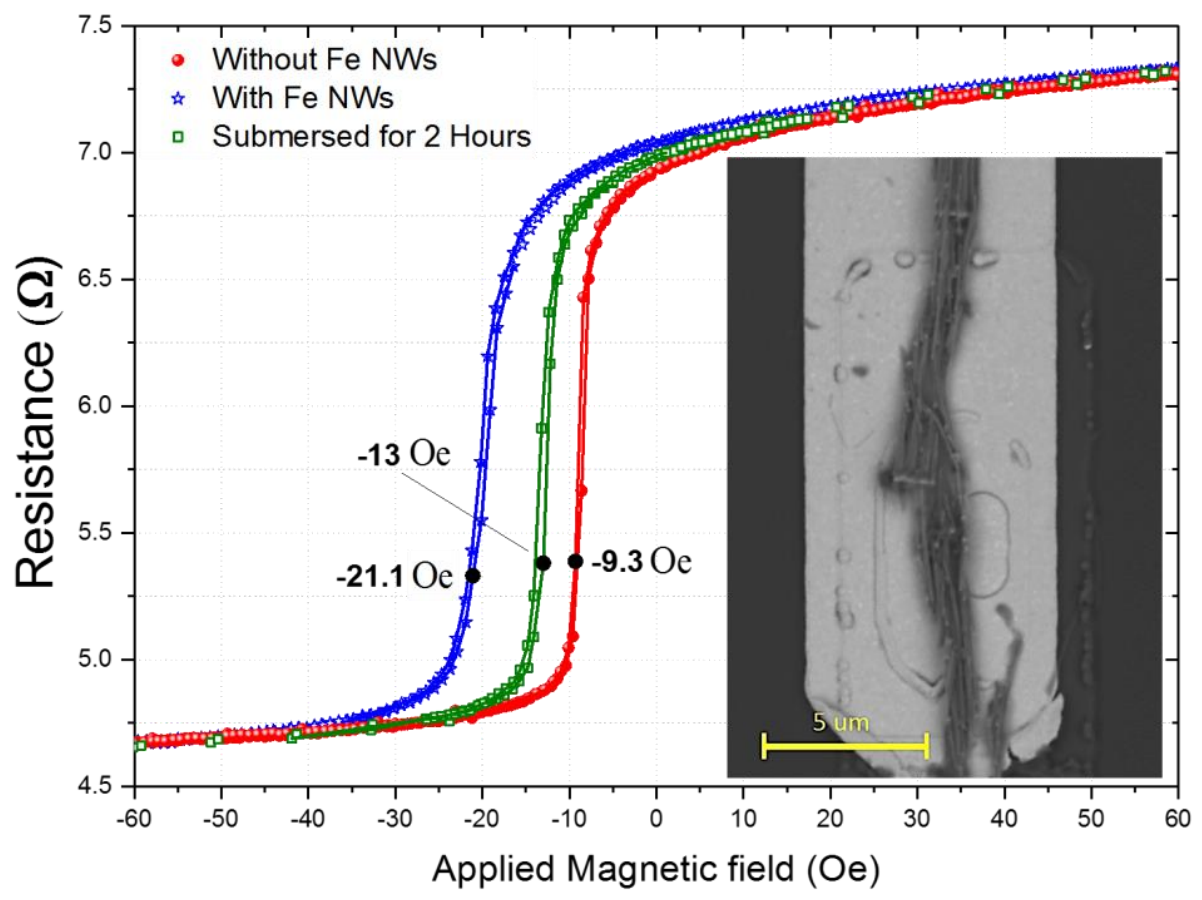

(b)

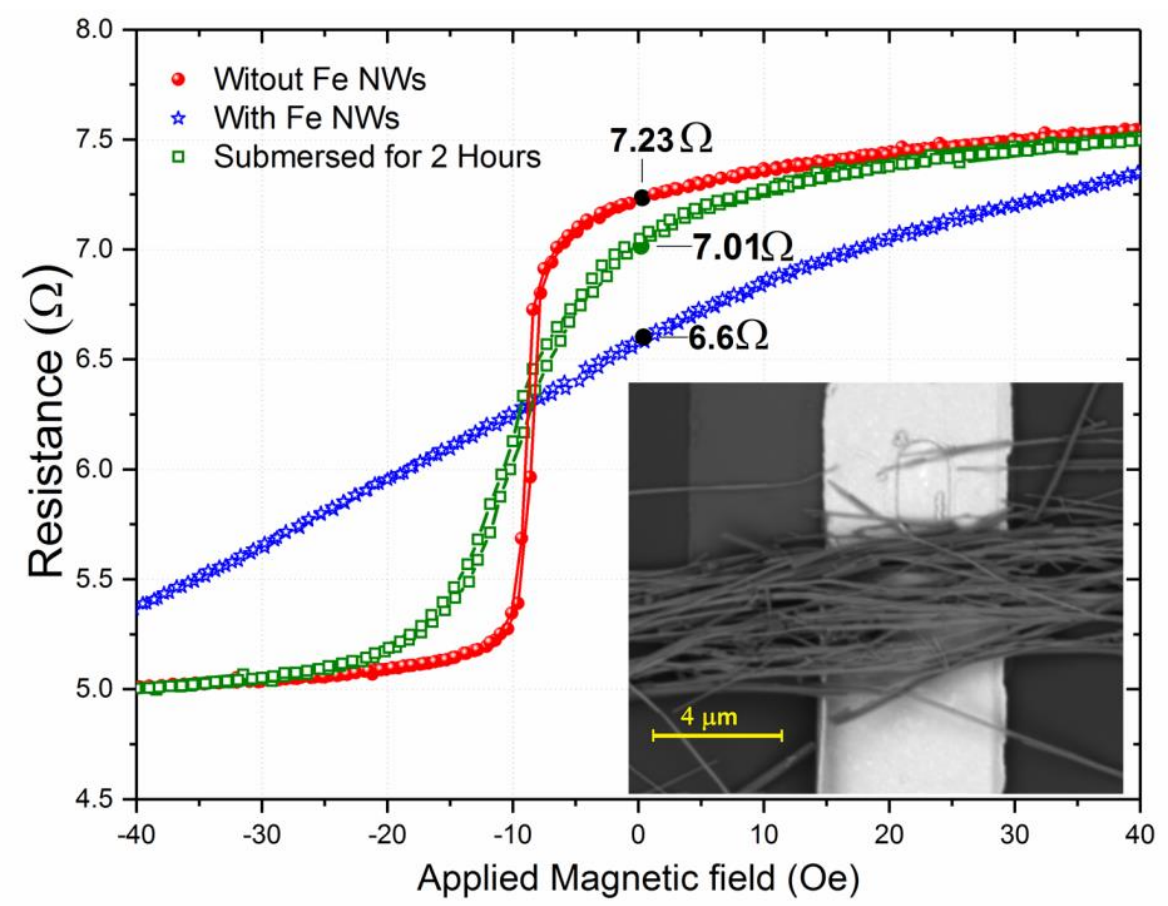

Figure 6. Characteristic curve of the MTJ sensor without NWs (red) and with Fe NWs on top of it (blue), and after submersing the MTJ with the NWs in the saline solution for 2 hours (green). (a) The NWs are aligned parallel to the easy axis of the MTJ (b) The NWs are aligned perpendicular to the easy axis of the MTJ. 


\section{WILEY-VCH}

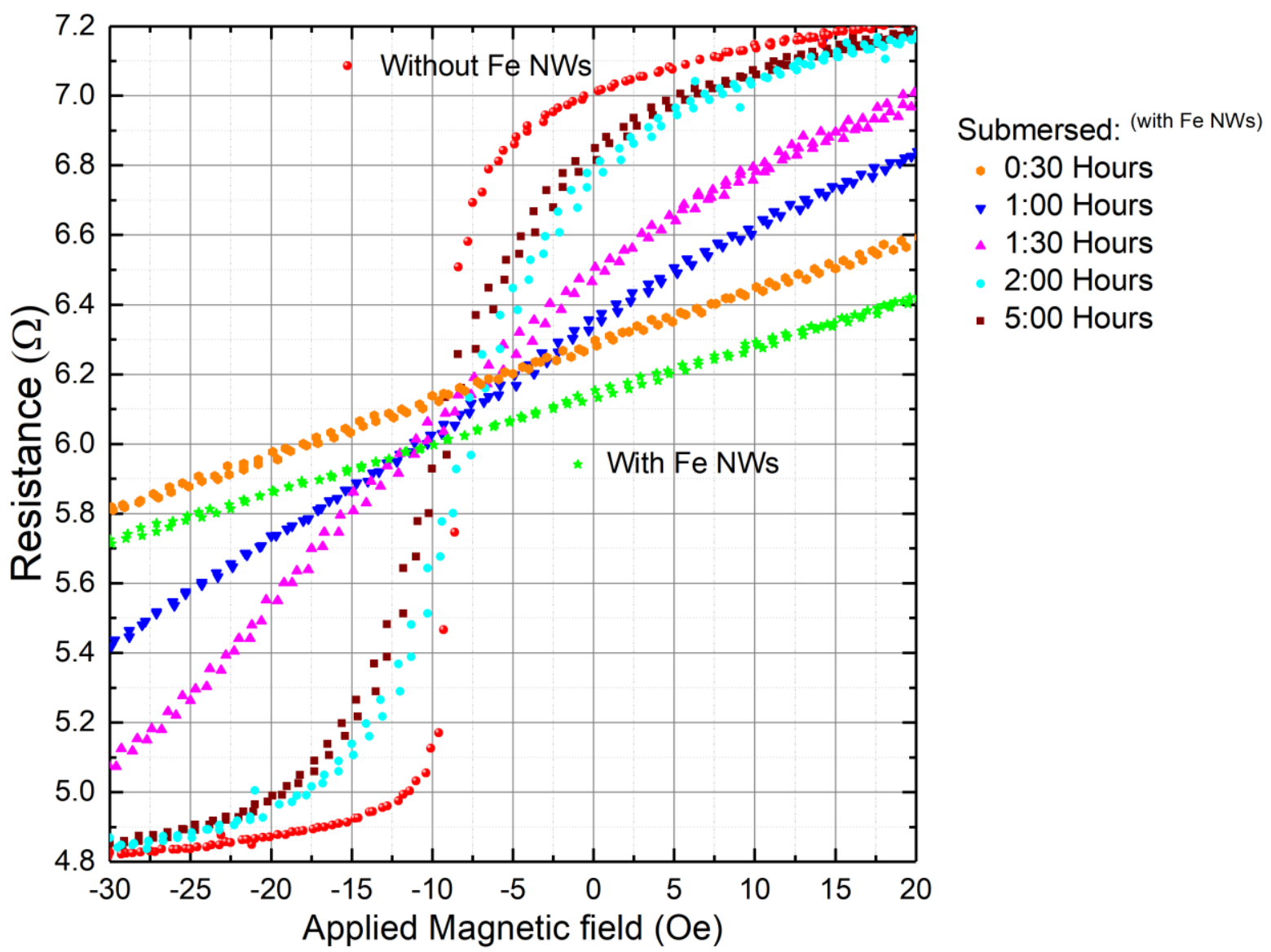

Figure 7. Characteristic curve of the MTJ sensor with and without NWs on top of it (aligned perpendicular to the easy axis), and when submersed in saline solution for up to 5 hours with the NWs. 
(a)

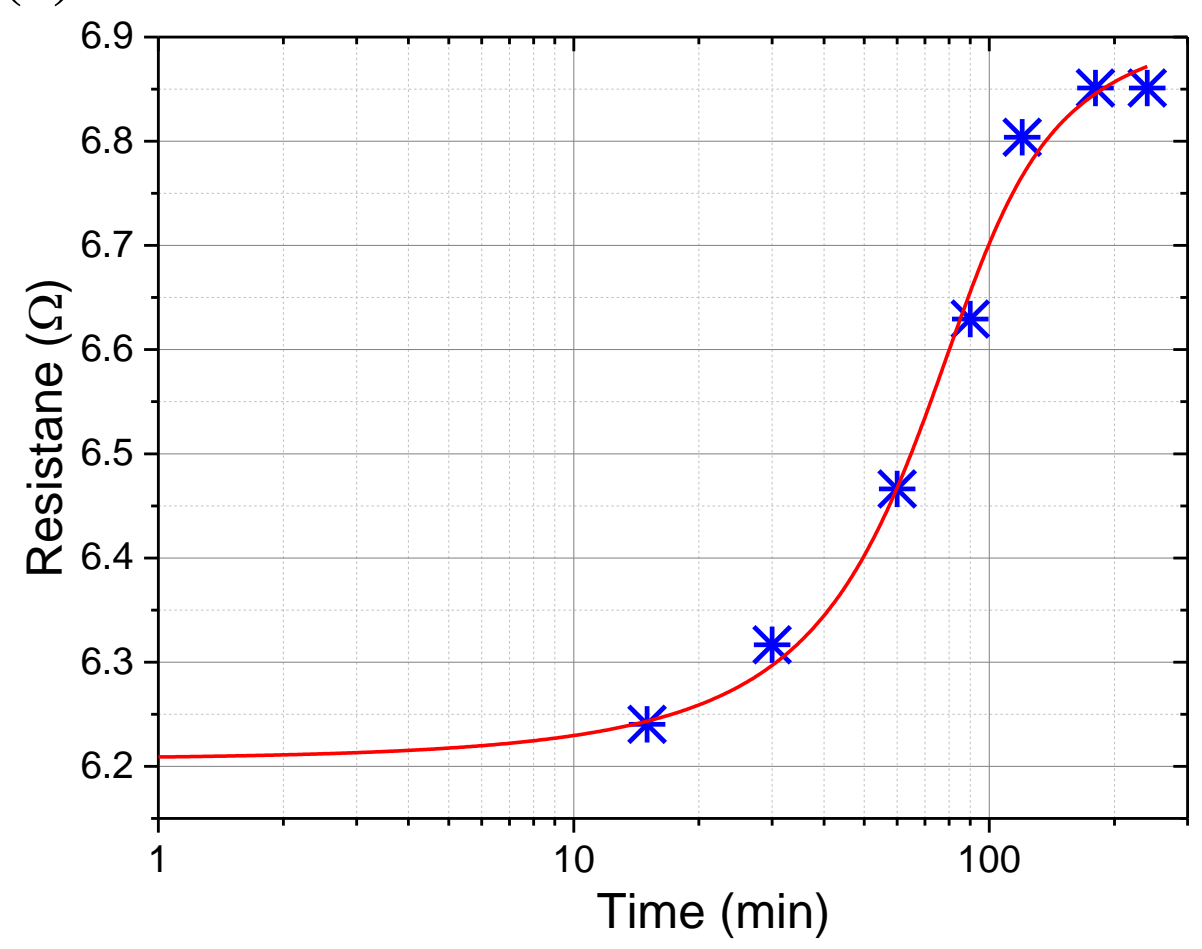

(b)

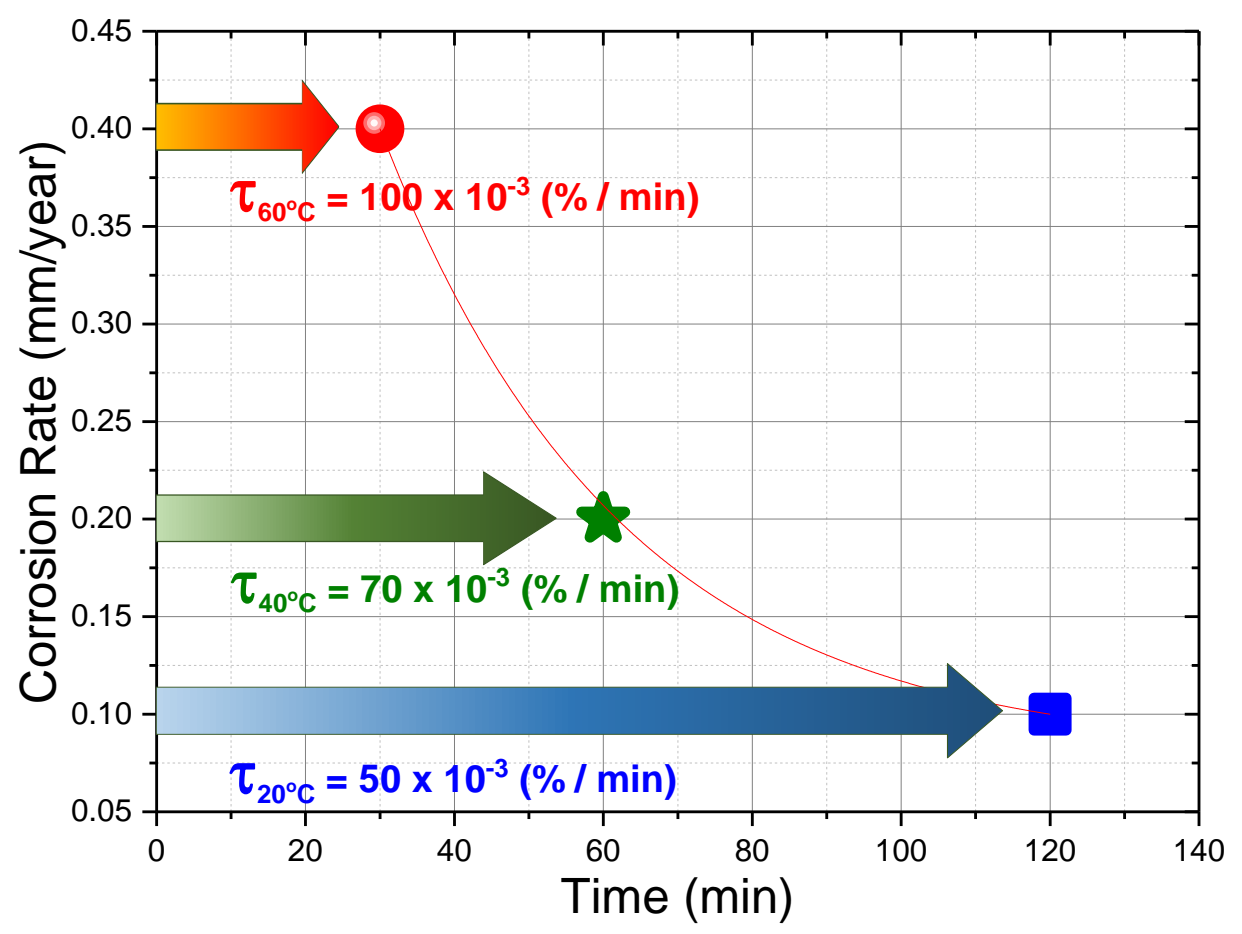

Figure 8. (a) Zero field resistance characteristic of the submersed corrosion sensor over time.

(b) The corrosion rates dependence on temperature. The sensitivities $\boldsymbol{\tau}_{\mathbf{2 0}}{ }^{\circ} \mathbf{C}, \boldsymbol{\tau}_{40}{ }^{\circ} \mathbf{C}$, and $\boldsymbol{\tau 6 0}^{\circ} \mathbf{C}$ are calculated at $20{ }^{\circ} \mathrm{C}, 40{ }^{\circ} \mathrm{C}$ and $60{ }^{\circ} \mathrm{C}$, respectively. 